\title{
HIGHLIGHTS
}

IBS

\section{Rifaximin provides effective and sustained relief of IBS symptoms}

Treatment with rifaximin, a minimally absorbed antibiotic, provides substantial symptomatic relief in patients with IBS without constipation. "Many drugs studied in IBS lead to improvement in more than one symptom, but with rifaximin all the symptoms were improved," comments Mark Pimentel of Cedars-Sinai Medical Center, CA, USA.

The gut microbiota is thought to have a role in the development of IBS, which led researchers to consider antibiotics as therapy for this condition. However, conventional antibiotics for IBS are associated with problems, such as a narrow antimicrobial spectrum and bacterial resistance.

In two, identically designed, phase III, randomized, double-blind trials, 1,258 patients who had IBS without constipation received either placebo or rifaximin $550 \mathrm{mg}$ three times daily for 2 weeks. Participants were followed up for another 10 weeks. Adequate relief of global IBS symptoms occurred in more patients in the rifaximin group than the placebo group (40.7\% versus $31.7 \%$ in the pooled data). In addition, increased rates of relief of IBS-related bloating, abdominal pain or discomfort and improved stool consistency were reported by the rifamixin group. Improvements in IBS-related symptoms were maintained throughout the follow-up period in more patients treated with rifaximin than placebo. The two groups had similar rates of adverse events.

"We are now investigating treatments directed at gut bacteria in constipationpredominant IBS and already have a double-blind study underway," says Pimentel.

Shreeya Nanda

Original article Pimentel, M. et al. Rifaximin therapy for patients with irritable bowel syndrome without constipation. N. Engl. J. Med. 364, 22-32 (2011) 